\title{
Dal fegato al muscolo: una rara malattia a esordio tardivo
}

\author{
Claudia Brusadelli ${ }^{*}$, Margherita Calia*, Silvia Fasoli ${ }^{* *}$, Serena Gasperini ${ }^{* * *}$ \\ "Scuola di Specializzazione di Pediatria, Università degli Studi Milano-Bicocca, Monza; ** UOC Pediatria, Mantova; \\ ${ }^{* * *}$ Clinica Pediatrica, Fondazione MBBM, Università degli Studi Milano-Bicocca, Monza
}

\begin{abstract}
Descriviamo il caso di un bambino di sette anni che, in seguito al riscontro incidentale di ipertransaminasemia agli esami ematochimici eseguiti per una linfoadenopatia, è sottoposto ad altri accertamenti bioumorali che mostrano anche un persistente, moderato incremento dei valori di CPK, supportando l'ipotesi di una patologia muscolare pur in assenza di sintomatologia specifica. Il percorso diagnostico sembra orientare verso una miopatia metabolica a esordio tardivo. Il riscontro occasionale di ipertransaminasemia, associata al rilievo di iperCPKemia in un bambino asintomatico o paucisintomatico, orienta la diagnosi differenziale, esclusa una malattia da accumulo epatico o infettiva virale, verso una miopatia complessa a esordio tardivo. Si pensa pertanto a una rara malattia metabolica che più frequentemente compare in epoca neonatale o nel piccolo lattante e inizia un percorso diagnostico che porterà alla diagnosi definitiva.
\end{abstract}

\section{We describe the case of a seven-year-old child who, following the incidental finding of hypertransaminasemia in the blood tests performed for a lymphadenopathy, is subjected to other blood tests that show a persistent moderate increase in CPK values, supporting the hypothesis of a muscle disease even in the absence of specific symptoms. \\ The diagnostic path seems to point towards a late-onset metabolic myopathy. \\ The occasional finding of hypertransaminases, associated with the finding of high CPK levels in an asymptomatic or paucisymptomatic child, orients the diagnostic path. Excluding hepatic storage disease or viral infectious disease, the differential diagnosis leads to a complex late-onset myopathy such as Pompe disease, a rare but important diagnosis whose prognosis could be dramatically improved by the enzyme replacement therapy.}

\section{La storia}

P. è un bambino di sette anni, terzogenito figlio di genitori non consanguinei, con anamnesi familiare, fisiologica e patologica remota mute. In seguito alla comparsa in apiressia di linfoadenopatia latero-cervicale e angolomandibolare bilaterale (diametro massimo $1,5 \mathrm{~cm}$ ) di aspetto infiammatorio e con cute sovrastante indenne, esegue un controllo bioumorale su indicazione del curante. Da tali accertamenti emerge un incremento dei valori di AST, ALT e LDH (AST 199 U/1 v.n. 13-40, ALT 189 U/1 v.n. 7-40 e LDH 1081 U/1 v.n. 120-246) con indici di flogosi lievemente aumentati (PCR 7,9 mg/dl v.n. <1) e sierologie per CMV, EBV e ADV negative per infezioni in atto. Per questi esami è pertanto inviato presso il pronto soccorso e ricoverato. All'ingresso il bambino appare in buone condizioni generali, all'obiettività si osserva solo la già descritta linfoadenopatia. Durante la degenza gli esami ematochimici confermano AST, ALT e $\mathrm{LDH}$ persistentemente elevati in assenza di indici di colestasi, gammaGT nella norma e con sintesi epatica conservata. Si osserva, inoltre, un moderato incremento dei valori di CPK, non prima determinati (valore massimo 1449 U/1 v.n. 46-171). Lecografia del collo conferma la presenza di formazioni linfonodali di aspetto reattivo. L'ecografia addome è nella norma.

A questo punto il quadro clinico generale è quello di un bambino di 7 anni in buone condizioni generali con una linfoadenopatia bilaterale del collo con grandezza massima di $1,5 \mathrm{~cm}$ associata a ipertransaminasemia $(\times 4)$ e incremento delle CPK. Il fegato si palpa all'arcata costale, di consistenza parenchimatosa. La milza è palpabile all'arco costale. Gli esami di primo livello, tesi a escludere infezioni in atto, sono negativi. Infatti, i test sierologici e molecolari per i principali agenti eziologici di epatite e miosite sono nella norma. Il bambino presenta tono e trofismo muscolare adeguati, osservabile solo un lieve deficit di forza del cingolo muscolare inferiore.

\section{La diagnosi e il decorso}

Sono pertanto passate in rassegna e scartate eziologie alternative:

- neoplastica. Sono nella norma: emocromo, striscio di sangue periferico, marker tumorali, ecografia di collo e addome, $\mathrm{Rx}$ torace;

- autoimmune: celiachia, $\mathrm{C} 3$ e C4, autoanticorpi negativi (ANA, AMA);

- malattia epatica da accumulo: quadro marziale, ceruloplasmina e cupremia, alfa1AT nei limiti;

- nell'ipotesi di una rara malattia metaboli$c a$, considerato il contemporaneo incremento delle transaminasi, è stato eseguito il dosaggio dell'attività dell'enzima alfa-1,4 glucosidasi su cartoncino Guthrie (DBS, dried blood spot) che risulta ridotto: $0,2 \mathrm{micromol} / \mathrm{L} / \mathrm{h}$ (cut-off $>2$ ). Il bambino è quindi inviato presso il nostro centro di malattie metaboliche nel sospetto di malattia di Pompe. La diagnosi è confermata dal dosaggio dell'attività dell'enzima alfa-1,4 glucosidasi su leucociti (0,19 UM v.n. $>0,35)$ e dall'analisi molecolare del gene codificante l'enzima alfa 1,4-glucosidasi (GAA) che evidenzia le seguenti mutazioni in eterozigosi: la variante patogenetica nota c. $-32-13 \mathrm{~T}>\mathrm{G}$ e la delezione nota c.2481+110_2646+$39 \operatorname{del} 538$.

P. è pertanto affetto da malattia di Pompe a esordio tardivo paucisintomatica al momento della diagnosi: la valutazione neuromuscolare evidenzia solo scapole alate bilaterali e un lieve deficit del cingolo muscolare inferiore con tuttavia un ottimo trofismo muscolare. La RM muscolare degli arti inferiori risulta nella norma e l'ecocardiogramma esclude un interessamento cardiaco. Gli accertamenti biochimici e molecolari sono estesi a tutto il nucleo familiare: il piccolo ha ereditato ciascuna delle due mutazioni dai genitori, risultati entrambi portatori perché anche il fratello maggiore risulta affetto. In considerazione della variabilità fenotipica della malattia e della possibile progressione del quadro cli- 
nico si propone alla famiglia di avviare per P. la terapia enzimatica sostitutiva. Il fratello maggiore è invece riferito a un centro di malattie metaboliche dell'adulto dove è confermata la diagnosi, supportata anche dall'esito della RM muscolare degli arti inferiori e dell'esame istologico della biopsia muscolare che sono compatibili con la malattia.

\section{Commento}

Il riscontro occasionale dell'incremento dei valori di CPK in corso di accertamenti per ipertransaminasemia di origine non epatica non è un evento raro in pediatria [1]. L'iter diagnostico inizia con la conferma del dato a distanza di tempo e a riposo, e con l'esclusione di cause note di iperCPKemia (Figura 1) [2]. Particolare attenzione deve essere posta alla storia famigliare (presenza di miopatie, suscettibilità all'ipertermia maligna, morti improvvise) e allo sviluppo muscolare del bambino oltre che a segni di scoliosi o deviazione del rachide [1]. Il percorso nel paziente pediatrico, in caso di valori moderati-alti e persistenti di CPK (>1000 U/L), prosegue in genere con l'analisi enzimatica e molecolare (esistono pannelli disponibili) e, meno frequentemente rispetto all'adulto, con l'elettromiografia e la biopsia muscolare. Lo spettro delle miopatie è tuttavia molto ampio e non ancora pienamente conosciuto, rendendo pertanto necessario nella maggior parte dei casi l'invio del bambino a un centro di riferimento per patologie neuromuscolari $[1,3]$. Tra le cause di miopatia metabolica, la malattia di Pompe è una patologia rara (incidenza della forma adulta di 1:60.000 nella popolazione europea), ma probabilmente sottostimata. La malattia di Pompe, anche nota come glicogenosi tipo II, è una patologia autosomica recessiva causata dalla mutazione del gene GAA che codifica per l'enzima alfa 1,4-glucosidasi o maltasi acida. Il deficit di questo enzima determina un accumulo di glicogeno lisosomiale nei muscoli scheletrici, respiratori e a livello cardiaco. La severità del quadro clinico è variabile in base all'età di esordio, al genotipo e al grado di coinvolgimento d'organo. Esistono due forme principali di malattia di Pompe: classica a esordio infantile (IOPD, infantile onset pompe disease) e late-onset (LOPD, late-onset pompe disease), anche se in realtà la patologia si caratterizza per un continuum fenotipico come per le altre malattie lisosomiali. Le principali caratteristiche cliniche sono riassunte nella Tabella 1.

La diagnosi può essere sospettata con il riscontro di una ridotta attività enzimatica dell'alfa-glucosidasi su DBS e richie-

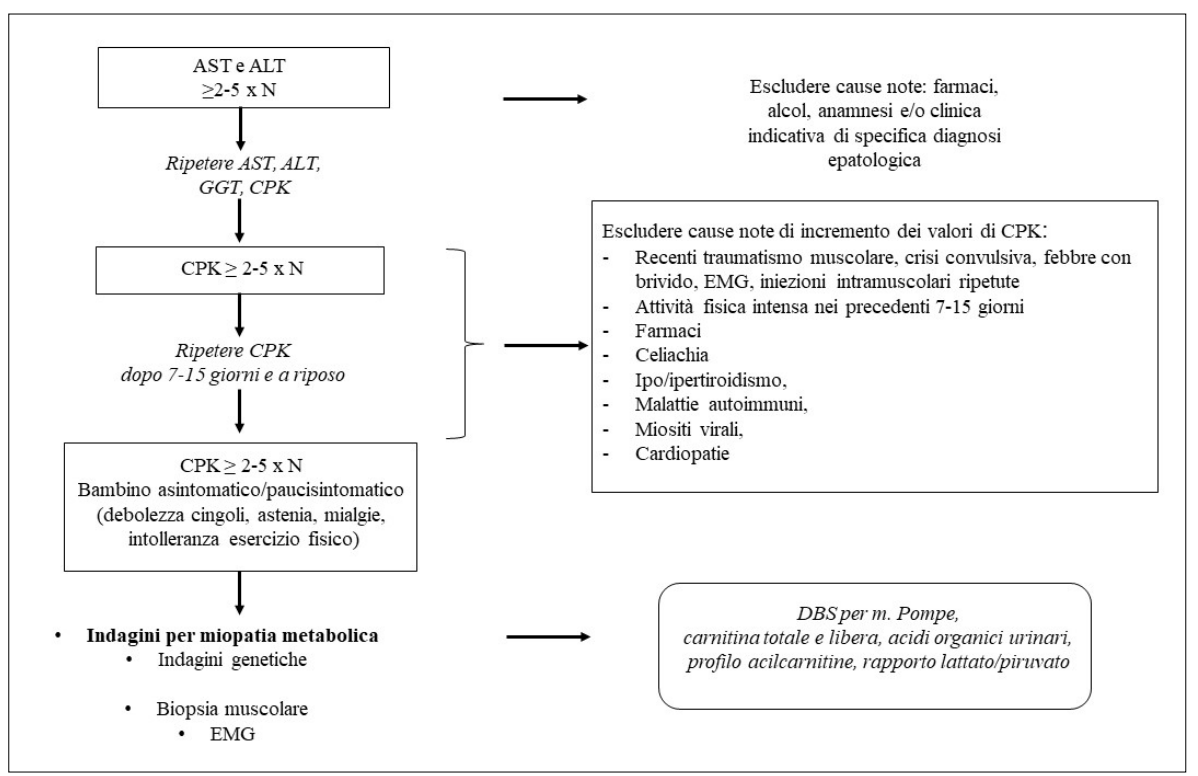

Figura 1. Flow chart diagnostica in caso di ipertransaminasemia suggestiva di iperCPKemia (da voci bibliografiche 2 e 4 modificate). AST, aspartato transaminasi; ALT, alanina transaminasi; CPK, creatin fosfato chinasi; EMG, elettromiografia; DBS, dried blood spot.

\begin{tabular}{|c|c|c|}
\hline FORME & $\begin{array}{l}\text { MALATTIA DI POMPE } \\
\text { CLASSICA A ESORDIO } \\
\text { INFANTILE (IOPD) }\end{array}$ & $\begin{array}{l}\text { MALATTIA DI POMPE } \\
\text { LATE-ONSET (LOPD) }\end{array}$ \\
\hline Esordio & $<12$ mesi & $>1-2$ anni $\rightarrow 60$ anni \\
\hline Attività enzima GAA & $<1 \%$ & $2-40 \%$ \\
\hline $\begin{array}{l}\text { Interessamento } \\
\text { cardiaco }\end{array}$ & $\begin{array}{l}\text { Cardiomiopatia ipertrofica, aritmie, } \\
\text { insufficienza cardiorespiratoria }\end{array}$ & Generalmente assente \\
\hline $\begin{array}{l}\text { Interessamento } \\
\text { muscolare }\end{array}$ & $\begin{array}{l}\text { Ipotonia severa progressiva } \\
\text { (floppy infant) }\end{array}$ & $\begin{array}{l}\text { Ipotonia variabile e progressiva } \\
\text { (miopatia prossimale dei cingoli, } \\
\text { scapole alate, in alcuni casi } \\
\text { evoluzione in ipotonia assiale } \\
\text { e insufficienza respiratoria) }\end{array}$ \\
\hline Sviluppo motorio & Ritardo motorio o regressione & Nella norma \\
\hline $\begin{array}{l}\text { Altre caratteristiche } \\
\text { cliniche }\end{array}$ & $\begin{array}{l}\text { Epatomegalia, macroglossia, } \\
\text { scarsa crescita }\end{array}$ & $\begin{array}{l}\text { Rara epatomegalia, rara } \\
\text { macroglossia, infezioni respiratorie }\end{array}$ \\
\hline Laboratorio & $\mathrm{CPK} \uparrow \uparrow, \mathrm{AST} \uparrow, \mathrm{ALT} \uparrow$ & $\mathrm{CPK} \uparrow, \mathrm{AST} \uparrow, \mathrm{ALT} \uparrow$ \\
\hline Outcome & $\begin{array}{l}\text { Exitus intorno a } 12 \text { mesi di vita } \\
\text { (in assenza di terapia enzimatica } \\
\text { sostitutiva) }\end{array}$ & Variabile \\
\hline
\end{tabular}

de conferma mediante l'analisi molecolare del gene GAA o il dosaggio dell'attività enzimatica in altro tessuto (per esempio i leucociti) [6]. Nel caso descritto P. è portatore della variante c.-32-13T $>\mathrm{G}$, legata alle forme a esordio in età adulta, e della delezione c.2481+110_2646+39de1538, solitamente riscontrata nella malattia classica infantile. In letteratura sono descritte associazioni delle due mutazioni in fenotipi a elevata variabilità clinica $[6,7]$. La prognosi della malattia di Pompe è significativamente migliorata grazie all'introduzione dell'ERT che consiste in somministrazioni endovenose ogni due settimane di enzima alglucosidasi alfa ricombinante umano. La ERT, di sicura efficacia nelle forme infantili, ne ha cambiato la curva di sopravvivenza permettendo di evitare o ritardare il ricorso alla ventilazione meccanica e ha garantito in molti bambini una discreta qualità di vita fino al raggiungimento della deambulazione. Nelle forme a esordio tardivo asintomatiche, la ERT mira a prevenire la comparsa delle manifestazioni cliniche e a rallentare l'evolutività dell'interessamento muscolare [6]. Tuttavia, l'avvio della ERT nelle forme a esordio tardivo viene valutato caso per caso, considerata la variabilità fenotipica della malattia adulta e i possibili rischi associati alla terapia. Sussiste, infatti, la possibilità di insorgenza di reazioni infusionali che sono più frequenti nelle forme infan- 
tili in pazienti cosiddetti CRIM (Cross Reactive Immunologic Material) negativi, che non hanno attività enzimatica residua e pertanto sviluppano una reazione anticorpale avversa all'enzima [8]. Inoltre, tale decisione deve essere concordata con la famiglia trattandosi di una terapia endovenosa periodica che si protrae per tutta la vita ed è difficilmente sospendibile. In ogni caso l'obiettivo della diagnosi precoce è prevenire i sintomi spesso nascosti o poco evidenti che possono slatentizzarsi, anche drammaticamente, in corso di episodi infettivi intercorrenti, soprattutto respiratori. Nel caso specifico l'età della diagnosi e la presenza di una mutazione associata alle forme infantili più severe hanno fatto propendere per la proposta di iniziare la terapia enzimatica.

\section{Conclusioni}

Dal caso e dalla letteratura abbiamo imparato che:

- l'ipertransaminasemia non è solo di origine epatica e muscolare per cui si dovrebbe sospettare una miopatia e dosare le CPK in caso di persistente incremento di AST e ALT con gammaGT nella norma. In alcune forme di malat- tia di Pompe late-onset il riscontro di ipertransaminasemia precede anche di anni l'iperCPKemia;

- l'iter diagnostico dell'iperCPKemia deve tenere in considerazione la malattia di Pompe, malattia di importante diagnosi tramite cartoncino Guthrie (DBS). Il DBS, analizzato mediante spettrometria di massa, è infatti un'indagine di semplice esecuzione e non invasiva (rispetto a una biopsia muscolare), si spedisce con posta ordinaria ai centri di riferimento per l'analisi e fornisce esiti in tempi rapidi;

- la malattia di Pompe non è al momento compresa nel pannello dello screening neonatale esteso (SNE) in tutto il territorio nazionale, ma è stata solo di recente introdotta nello SNE come progetto pilota in alcune Regioni. In considerazione dell'importanza della diagnosi precoce ai fini dell'inizio del trattamento in epoca presintomatica, soprattutto nelle forme a esordio infantile, la malattia di Pompe è una patologia candidabile all'inserimento nel pannello SNE.
1. Sabouraud P, Cuisset JM, Cances C, et al. Stratégie diagnostique devant une hyperCKémie chez l'enfant. Arch Pediatr 2009 Jun;16(6):678-80.

2. Morandi L, Angelini C, Prelle A, et al. High plasma creatine kinase: review of the literature and proposal for a diagnostic algorithm. Neurol Sci 2006 Nov;27(5):303-11.

3. Rubegni A, Malandrini A, Dosi C, et al. Next-generation sequencing approach to hyperCKemia: A 2-year cohort study. Neurol Genet 2019 Aug 16;5(5):e352.

4. Al-Ghamdi F, Darras BT, Ghosh PS. Spectrum of Neuromuscular Disorders With HyperCKemia From a Tertiary Care Pediatric Neuromuscular Center. J Child Neurol 2018 May;33(6):389-96.

5. Kishnani PS, Steiner RD, Bali D, et al. Pompe disease diagnosis and management guideline. Genet Med 2006 May;8(5):267-88. 6. Leslie N, Bailey L. Pompe Disease. In Adam MP, Ardinger HH, Pagon RA, et al. (a cura di). GeneReviews. University of Washington, 2007.

7. Reuser AJJ, van der Ploeg AT, Chien YH, et al. GAA variants and phenotypes among 1,079 patients with Pompe disease: Data from the Pompe Registry. Hum Mutat 2019 Nov;40(11):2146-2164.

8. Cupler EJ, Berger KI, Leshner R,T et al. Consensus treatment recommendations for late-onset Pompe disease. Muscle Nerve 2012 Mar;45(3):319-33.

\section{Conversare a cinque mesi di età: si può e si deve}

Abbiamo già raccontato che il turn taking fa bene alla salute (Quaderni acp 2019;3:134): bambini che fanno più turni di conversazione con i genitori all'età di 18-24 mesi presentano un migliore 01 a 9-14 anni [1]. Chiacchierare con un bambino di 2 anni si può fare, ma instaurare una conversazione con un bambino di 5 mesi è possibile? In uno studio sperimentale i ricercatori dell'università di Stanford hanno arruolato 151 diadi mamma-bambino di età 5-8 mesi. 99 diadi hanno completato la prima parte dello studio che prevedeva la registrazione audio delle conversazioni rivolte al bambino tramite un dispositivo indossato dal piccolo e collegato a un registratore audio in grado di misurare e classificate tutte le parole che vengono rivolte al bambino, distinguendo anche di chi è la voce; utilizzando questi dati, i ricercatori sono stati in grado di calcolare la quantità di conversazioni che i bambini hanno intrattenuto con gli adulti in un ambiente naturale. Infine, di 51 lattanti si è riusciti a ottenere uno studio di risonanza magnetica funzionale cerebrale (fMR) durante il sonno. Nelle diadi dove era presente una più accentuata conversazione (frasi rivolte al bambino $\mathrm{e}$ risposte tramite vocalizzi o lallazioni) la fMR rilevava una modificazione nelle attivazioni delle connessioni di una rete temporale posteriore che coinvolgeva la corteccia uditiva e il giro temporale superiore bilaterale e una rete frontotemporale che coinvolgeva il giro frontale inferiore sinistro; in altre parole, le conversazioni del genitore rivolte al bambino e la quantità di interazioni vocali del lattante verso il genitore a questa età hanno permesso di identificare due particolari reti di connessione funzionale della corteccia implicate nella comprensione del linguaggio, indipendentemente dallo stato socioeconomico [2]. In particolare questo studio ha evidenziato che la rete frontotemporale, che si ritiene si sviluppi verso i 2 anni di età, nei lattanti che intrattengono più turni di conversazione è già rilevabile alla fine del primo semestre.

1. Mendelsohn AL, Klass P. Early Language Exposure and Middle School Language and IO: Implications for Primary Prevention. Pediatrics 2018 Oct;142(4)::e20182234.

2. King LS, Camacho MC, Montez DF, et al. Naturalistic Language Input is Associated with Resting-State Functional Connectivity in Infancy. J Neurosci 2021 Jan 20;41(3):424-34. 